\title{
Nuevos registros para la herpetofauna del departamento de Lima, descripción del renacuajo de Telmatobius rimac Schmidt, 1954 (Anura: Ceratophrydae) y una clave de los anfibios
}

\author{
New records for the herpetofauna of Lima, description of Telmatobius rimac \\ Schmidt, 1954 tadpole (Anura: Ceratophrydae) and a key to the amphibians
}

\section{César Aguilar', Mikael Lundberg², Karen Siu-Ting ${ }^{1}$ y María Elena Jiménez}

${ }^{1}$ Museo de Historia Natural, Universidad Nacional Mayor de San Marcos. Av. Arenales 1256, Jesús María, Apartado 14-0434, Lima, Perú.

${ }^{2}$ Staatliche Naturhistorische Sammlungen Dresden, Museum für Tierkunde, Königsbrücker Landstrasse 159, D-01109 Dresden, Germany

Email César Aguilar: caguilarp@unmsm.edu.pe

Presentado: $\quad 13 / 07 / 2007$ Aceptado: $\quad 12 / 09 / 2007$

\section{Resumen}

En el presente trabajo la larva de Telmatobius rimac es descrita y ocho nuevos registros de anfibios y reptiles para el departamento de Lima son dados a conocer. Los nuevos registros de anfibios para Lima son Gastrotheca peruana y Pleurodema marmorata. Los nuevos registros de reptiles son las lagartijas Phyllodactylus gerrhopygus y Ameiva edracantha, y las serpientes Leptotyphlops tricolor, Philodryas tachymenoides, Sibynomorphus vagus y Tantilla capistrata. Con este estudio la herpetofauna presente en el departamento de Lima queda compuesta de 7 anfibios y 33 reptiles. Se proporciona una clave de identificación para los anfibios y una lista actualizada de la herpetofauna del departamento de Lima.

Palabras clave: Telmatobius rimac, renacuajo, descripción, herpetofauna, Lima.

\section{Abstract}

The tadpole of Telmatobius rimac is described and eight species of amphibians and reptiles are reported for the first time in Departamento de Lima. New amphibians records are Gastrotheca peruana and Pleurodema marmorata. New reptile records are the lizards Phyllodactylus gerrophygus and Ameiva edracantha, and the snakes Leptotyphlops tricolor, Philodryas tachymenoides, Sibynomorphus vagus and Tantilla capistrata. With this study, the herpetofauna of Lima is composed of 7 amphibians and 33 reptiles. An identification key for amphibians and an updated list of the herpetofauna for Departamento de Lima is provided.

Keywords: Telmatobius rimac, tadpole, description, herpetofauna, Lima.
En particular, los estudios taxonómicos y nuevos registros de distribución para los anfibios y reptiles del departamento de Lima han recibido relativamente poca atención en los últimos años. Los listados preliminares para Perú de Rodríguez et al. (1993) y Carrillo e Icochea (1995) reportaron en el departamento de Lima 4 especies de anfibios y 25 de reptiles respectivamente. Icochea (1998) presenta una lista de la herpetofauna del departamento de Lima constituida por 4 especies de anfibios, 15 lagartijas y 10 serpientes. Icochea (1998) menciona que Colostethus littoralis Péfaur, 1984; y Macropholidus ruthveni Noble, 1921; son introducidas aunque pueden ser encontrados en estado libre. Además, Icochea (1998) no incluye a Stenocercus chrysopygus Boulenger, 1900 aunque Carrillo e Icochea (1995) ya la habían listado previamente. Tello (1998) proporcionó una clave para las lagartijas de departamento de Lima, en la que incluyó a M. ruthveni y $S$. chrysopygus, y sugiere que probablemente las poblaciones de Phyllodactylus reissii Peters, 1862 sean introducidas.

Liolaemus walkeri (Shreve, 1938), Microlophus koepckeorum (Mertens, 1956), Philodryas simonsii (Boulenger, 1900) y Tachymenis peruviana Wiegmann, 1834 ya estaban reportados para el departamento de Lima, pero no mencionados en los listados anteriores. L. walkeri fue reportada en Ticlio (Shrewe, 1938) y M. koepckeorum en Ancón (Henle y Ehrl, 1991). Del mismo modo, T. peruviana ya había sido registrada por Walker (1945) para Yauyos y Philodryas simonsii (Boulenger, 1900) ya había sido reportada por Thomas (1977) para Huarochri (muy probablemente Huarochirí).

La presencia de Bothrops roedingeri Mertens, 1942 en el departamento de Lima fue indicado en Pesantes-Segura (2000), algo que también fue adoptado por Campbell y Lamar (2004). Además Aguilar y Lundberg (2005) registraron a Telmatobius carrillae Morales, 1988 para el departamento de Lima, aumentando a 5 el número de anfibios (Bufo limensis Werner, 1901, Bufo spinulosus Wiegmann, 1834, C. littoralis, T. carrillae y $T$. rimac).

Los objetivos de este estudio son presentar los nuevos registros de anfibios y reptiles para el departamento de Lima, describir la larva de T. rimac, proporcionar una clave para los renacuajos y anfibios postmetamórficos y una lista actualizada de los anfibios y reptiles de departamento de Lima.

\section{Materiales y métodos}

El número de especimenes y estadios larvales examinados, así como sus localidades de las especies reportadas en este trabajo, se detallan en la Tabla 1. Las coordenadas geográficas y las elevaciones de los especimenes fueron registrados con un GPS 72 Garmin. Los especímenes fueron fijados en formalina al $10 \%$ y están depositados en el Departamento de Herpetología, Museo de Historia Natural de San Marcos (MUSM), Lima. Los especimenes fueron identificados en base a literatura her- 
Tabla 1. Especimenes examinados.

\section{Bufo limensis}

MUSM 6737-6739: Río Huaura, provincia Huaura, departamento Lima; MUSM 6763: San Mateo, provincia Huarochirí, departamento Lima; MUSM 6742 Lunahuaná, provincia Cañete, departamento Lima; MUSM 6752-6753: Atocongo, provincia Lima, departamento Lima; MUSM 6745-6746, MUSM 6748-6749: Chosica, provincia Lima departamento Lima; MUSM 17987 (larvas): Barba Blanca, provincia Huarochirí, departamento Lima; MUSM 19231 (larvas): río Pisco, provincia Pisco, departamento Ica; MUSM 9416-9417: Marcahuasi, provincia Huarochirí, departamento Lima.

Bufo spinulosus

MUSM 9408-9414: Caza Pato, provincia Pasco, departamento Pasco MUSM 23391: Yauricocha, provincia Yauyos, departamento Lima; MUSM 23393-23394: Carhuamayo, provincia Junín, departamento Junín; MUSM 16992 (larvas): Accochaca, provincia Canta, departamento Lima.

Colostethus littoralis

MUSM 4257, 4267: Barranco, provincia Lima, departamento Lima; MUSM 4270-4271: Huaylas, provincia Huaylas, departamento Ancash; MUSM 20283-20286: San Miguel, provincia Huarmey, departamento Ancash.

Gastrotheca peruana

MUSM 19668: km 15,7 en la carretera Cajatambo - Oyon, provincia Cajatambo, departamento Lima; MUSM 24828-24841, MUSM 23561 (larvas): km 4,1 de la carretera Cajatambo - Oyon, provincia Cajatambo, departamento Lima; MUSM 6627-6655: Huaychopampa, departamento de Ancash.

\section{Pleurodema marmorata}

MUSM 19669-19675: Ticlio, provincia Huarochirí, departamento de Lima; MUSM 20416-20421: Carpa, provincia Recuay, departamento Ancash; MUSM 7118 (larvas): Laguna de Paca, provincia Jauja, departamento Junín.

\section{Telmatobius carrillae}

MUSM 19509-19516, MUSM 19522-19523, MUSM 19524 (larvas): Ticlio provincia Huarochirí, departamento Lima; MUSM 5635-5638, MUSM 5641-5648, MUSM 5621 (larvas): Chocopitec, provincia Antonio Raimondi, departamento Ancash; MUSM 19525-19536: Oyon, provincia Oyon, departamento Lima

\section{Telmatobius rimac}

MUSM 19501-19504, 19625 (larvas): Alis, provincia Yauyos, departamento Lima; MUSM 19640-19649: Canta, provincia Canta, departamento Lima; MUSM 19229 (larvas), MUSM 19626 (larvas): Obrajillo, provincia Canta, departamento Lima; MUSM 19627 (larvas): Acochaca, provincia Canta, departamento Lima.

\section{Phyllodactylus gerrhopygus}

MUSM 19323: La Fortaleza, Imperial, provincia Cañete, departamento Lima; MUSM 5080: provincia Pisco, departamento Ica; MUSM 5081-5082: provincia Tacna, departamento Tacna; MUSM 5083, 5149: Arequipa, provincia Arequipa, departamento Arequipa.

Ameiva edracantha

MUSM 19667: Pativilca, provincia Barranca, departamento Lima; MUSM 13655-13657: provincia Trujillo, departamento La Libertad; MUSM 13662: Chicama, provincia Trujillo, departamento La Libertad.

\section{Philodryas tachymenoides}

MUSM 25353, 23348: Cajatambo, provincia Cajatambo, departamento Lima; MUSM 23479: Oyon, provincia Oyon, departamento Lima; MUSM 23454, MUSM 23469: Alis, provincia Yauyos, departamento Lima; MUSM 24858: Chaucayan, provincia Recuay, departamento Ancash;MUSM 25400, 25419: Cajacay, provincia Recuay, departamento Ancash.

\section{Sibynomorphus vagus}

MUSM 19676: Urapuris, Cajatambo, provincia Cajatambo, departamento Lima; MUSM 20583: Cajacay, provincia Recuay, departamento Ancash; MUSM 2660, 3395: Huaraz, provincia Huaraz, departamento Ancash; MUSM 2687, 2662, 3089: Huayllas, provincia Huayllas, departamento Ancash.

Tantilla capistrata

MUSM 19680: Tunan, Paramonga, provincia Barranca, departamento Lima; MUSM 25352, MUSM 25355, MUSM 25388-25390: Chaucayan, provincia Recuay, departamento Ancash. petológica (Duellman y Fritts, 1972; Duellman y Veloso, 1977; Wassersug y Duellman, 1984; Vellard, 1960; Dixon y Huey, 1970; Wilson, 1999; Peters et al., 1986) y comparándolos con la colección científica del Departamento de Herpetología del MUSM. Los estadios de los renacuajos fueron determinados siguiendo a Gosner (1960) (Tabla 2). Las medidas de los renacuajos fueron tomadas con un ocular micrométrico hasta el $0,01 \mathrm{~mm}$ más cercano y los dibujos se hicieron con asistencia de un microscopio estereoscópico Nikon SMZ 800 con cámara lúcida incorporada. La terminología de los caracteres larvales sigue a Altig y McDiarmid (1999). Las variables morfométricas usadas en la descripción de las larvas son (las abreviaciones entre paréntesis): longitud del cuerpo (LC), longitud total (LT), ancho del cuerpo (ANC), altura del cuerpo (ALC), diámetro del ojo (DO), distancia interorbital (DIO), distancia internarial (DIN), distancia hocico narina (DHN), distancia narina ojo (DNO), distancia hocico espiráculo (DHE), altura cola muscular en su inicio (ALCOM), altura máxima de la cola (ALCO), ancho de la cola muscular (ANCOM), ancho del disco oral (ANDO).

\section{Resultados}

\section{Nuevos registros para la herpetofauna del depar- tamento de Lima}

\section{ANURA}

\section{Gastrotheca peruana (Boulenger, 1900)}

Un macho de G. peruana (MUSM 19668) fue encontrado debajo de una piedra al costado de una pequeńa quebrada en el km 15,7 en la carretera Cajatambo - Oyon, Distrito Cajatambo, Provincia Cajatambo, a $4076 \mathrm{~m}$ de altitud. Se encontraron abundantes renacuajos y postmetamórficos de la misma especie cerca del $\mathrm{km} 4,1$ de la carretera Cajatambo - Oyon a $3.660 \mathrm{~m}$ de altitud, en un charco usado como fuente de agua para ganado. Este registro extiende el área de distribución aproximadamente $35 \mathrm{~km}$ al sureste de Chiquián, departamento de Ancash (Duellman y Fritts, 1972).

\section{Pleurodema marmorata (Duméril \& Bibron, 1841)}

Varios ejemplares (MUSM 19669, 19670, 19671, 19672, 19673, 19674 y 19675) de P. marmorata fueron encontrados en Ticlio con las coordenadas S 11³5'58.5”, W 076¹'36.6”, a $4.818 \mathrm{~m}$ de altitud, en el distrito de Chicla, provincia Huarochirí. $P$. marmorata ha sido reportado en la vertiente occidental de los Andes en los departamentos de Ancash (Lehr, 2002) y Arequipa (Pefaur et al., 1978), pero este es el primer registro para el departamento de Lima.

\section{SQUAMATA}

\section{Phyllodactylus gerrhopygus (Wiegmann, 1834)}

Un juvenil de P. gerrhopygus (MUSM 19323) fue encontrado debajo de una piedra en un talud al este del pueblo La Fortaleza, a $200 \mathrm{~m}$ de altitud, en el distrito de Imperial, provincia de Cañete. El talud era de arena y grava con grupos de piedras de mayor tamaño y parches de vegetación de Tillandsia spp. Este registro extiende el área de distribución aproximadamente $40 \mathrm{~km}$ Noroeste de Jaguay, departamento de Ica (Dixon y Huey, 1970). 


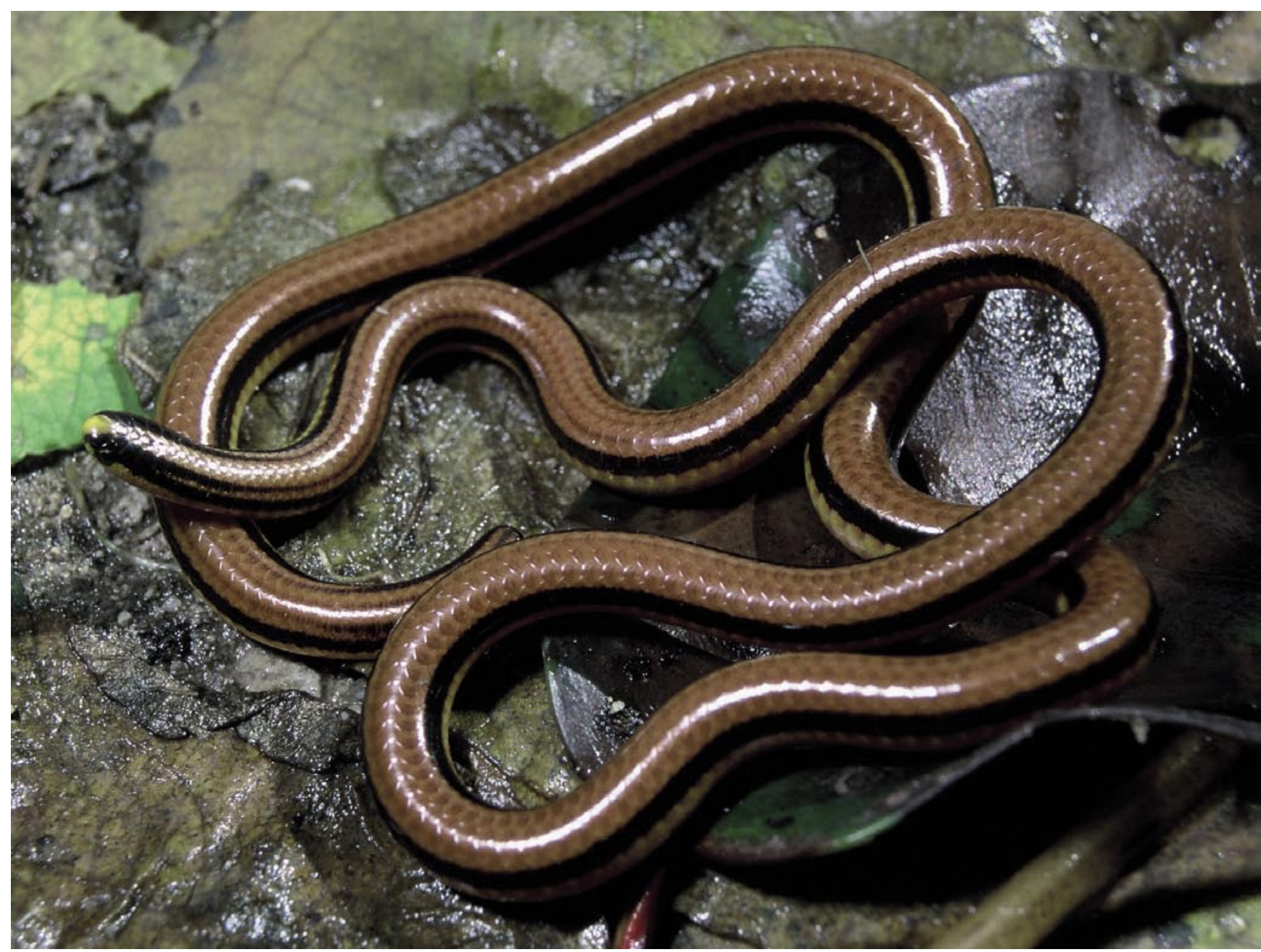

Figura 1. Leptotyphlops tricolor. Nuevo registro para el departamento de Lima.

\section{Ameiva edracantha Bocourt, 1874}

Un individuo de $A$. edracantha (MUSM 19667) fue encontrado en las Lomas de Paca con coordenadas S 10³7'50,0”, W 077043'34,0", a $521 \mathrm{~m}$ de altitud, en el distrito de Pativilca, provincia Barranca. Este registro extiende el área de distribución aproximadamente $65 \mathrm{~km}$ al suroeste de Cajacay, departamento de Ancash (Lehr y Aguilar, 2000).

\section{Philodryas tachymenoides (Schmidt \& Walker, 1943)}

P. tachymenoides se registró en varias localidades del departamento de Lima. En el norte (MUSM 25353) desde Cajatambo, a $3350 \mathrm{~m}$ de altitud, distrito de Cajatambo, provincia Cajatambo, y en el sur (MUSM 23454) hasta Alis, a 3300 m de altitud, distrito de Alis, provincia Yauyos. Estos primeros registros para el departamento de Lima están llenando un vacío de $700 \mathrm{~km}$ entre Cajacay en el departamento de Ancash (Lehr, 2002) y Atiquipa en el departamento de Arequipa (Zeballos et al., 2000). Esto indicaría que esta especie tiene una distribución geográfica continua en la vertiente occidental de los Andes desde el centro y sur de Perú hasta el norte de Chile (Donoso-Barros, 1966)

\section{Sibynomorphus vagus (Jan, 1863)}

Un ejemplar de la especie S. vagus (MUSM 19676) fue obtenido de un comunero del pueblo de Cajatambo. La serpiente fue colectada en sus terrenos de cultivo y estaba en una botella con alcohol siendo usado como remedio. La localidad de colecta de la serpiente fue en Urapuris, en el kilometro 4,5 de la carretera Cajatambo-Lima, distrito Cajatambo, provincia Cajatambo, a $3270 \mathrm{~m}$ de altitud. Este registro extiende el área de distribución aproximadamente $60 \mathrm{~km}$ al sureste de Cajacay, departamento de Ancash (Lehr, 2002).

\section{Tantilla capistrata Cope, 1876}

Partes de un ejemplar de T. capistrata (MUSM 19680) fueron obtenidos de un comunero. La serpiente fue colectada en su terreno de cultivo cerca de Tunan, distrito Paramonga, provincia Barranca y las partes fueron colocadas en una botella con alcohol junto con serpientes de otras especies que eran usados como remedio. Este registro extiende el área de distribución aproximadamente $75 \mathrm{~km}$ al sur de Huambo, departamento de Ancash (Lehr, 2002).

\section{Leptotyphlops tricolor Orejas-Miranda \& Zug, 1974}

Tres ejemplares fueron encontrados el 13 de abril de 2001 entre Lucumani y Pampa de Zárate, distrito de San Bartolomé, provincia de Huarochirí, aproximadamente a 2,700 m de altitud. El ejemplar más grande midió $270 \mathrm{~mm}$ (Fig. 1). Los tres individuos fueron encontrados debajo de la misma piedra en un día lluvioso. Después de ser fotografiados e identificados, los especimenes se extraviaron. Este registro extiende el área de 

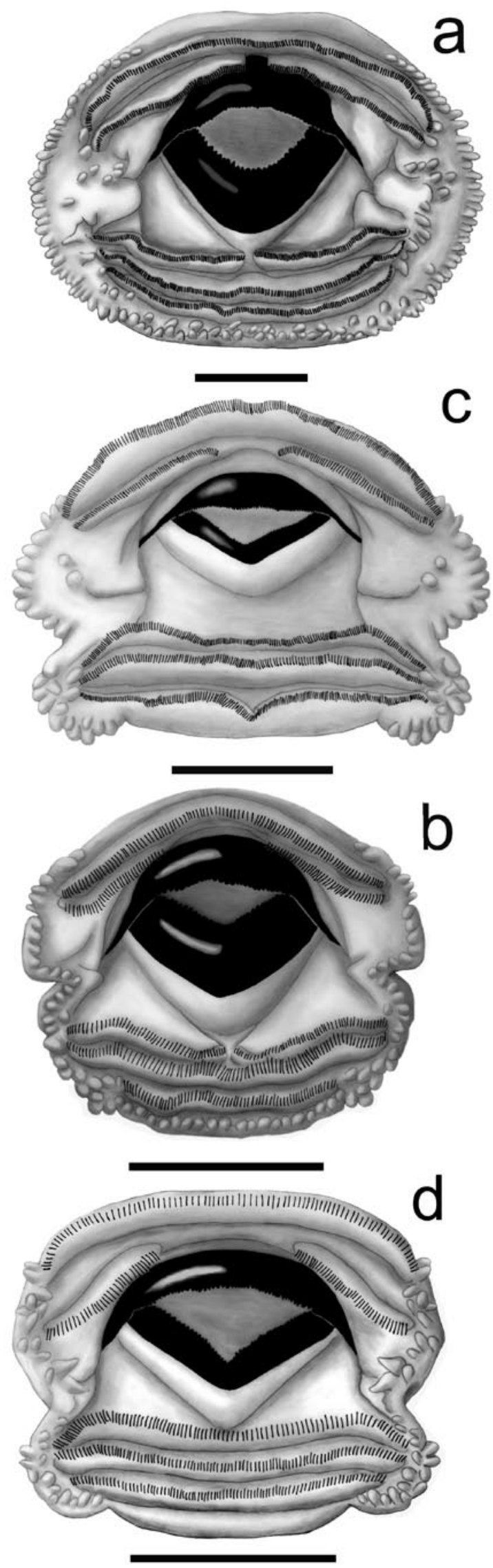

Figura 2. (a) Disco oral de la larva de Telmatobius rímac.(b) Disco oral de la larva de Pleurodema marmorata. (c) Disco oral de la larva de Bufo limensis. (d) Disco oral de la larva de Bufo spinulosus. Barra en la parte inferior de cada dibujo $=1 \mathrm{~mm}$.

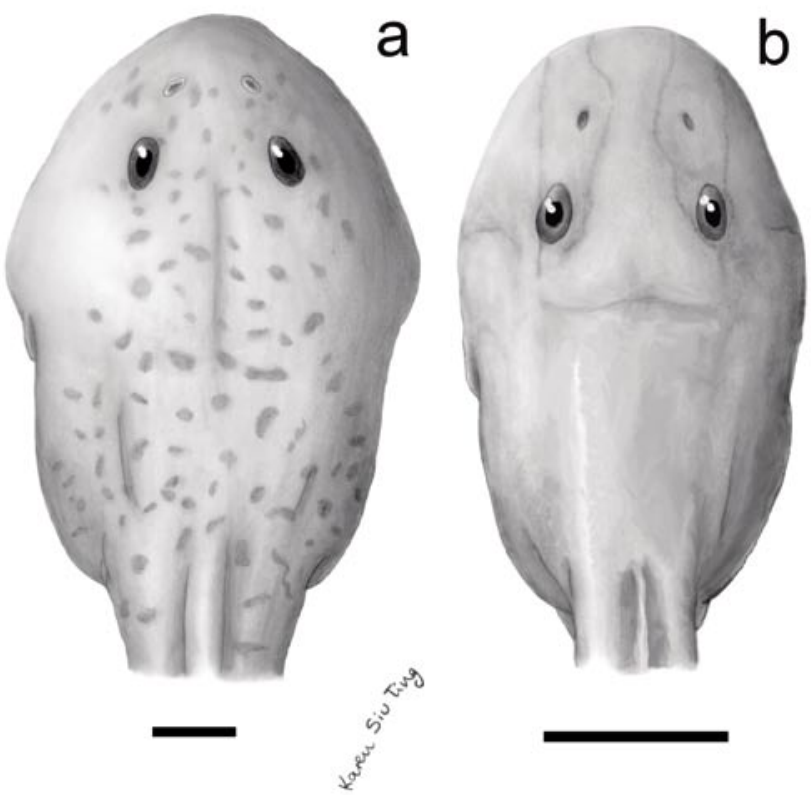

Figura 3. (a) Vista dorsal del cuerpo de la larva de Telmatobius carrillae. (b) Vista dorsal del cuerpo de la larva de Telmatobius rímac. Barra de escala $=5 \mathrm{~mm}$.

distribución aproximadamente $240 \mathrm{~km}$ al sureste de Huaráz, departamento de Ancash (Zug, 1977).

\section{Descripción del renacuajo de Telmatobius rimac}

Los renacuajos de T. rimac son asignables al gremio ecomorfológico de larvas exotróficas incluidas en la Sección I, grupo B7 de Altig y Johnston (1989). La descripción se basa en un espécimen en estadio 27 y las mediciones de las larvas examinadas se muestran en la Tabla 2.

Largo del cuerpo $11,10 \mathrm{~mm}$, longitud total $33,00 \mathrm{~mm}$. Cuerpo ovoide en vista dorsal; altura máxima del cuerpo $4,70 \mathrm{~mm}$, ancho máximo del cuerpo $6,05 \mathrm{~mm}$. Hocico redondeado en vista dorsal (Fig. 3b) y lateral. Ojos de posición dorsal, orientados lateralmente, diámetro 1,30 mm. Distancia interorbital 3,20 mm. Narinas externas de posición dorsal, ubicadas a un tercio de la distancia de los ojos al hocico. Espiráculo sinistral y lateral, abertura del espiráculo orientado posterodorsalmente, pared interna
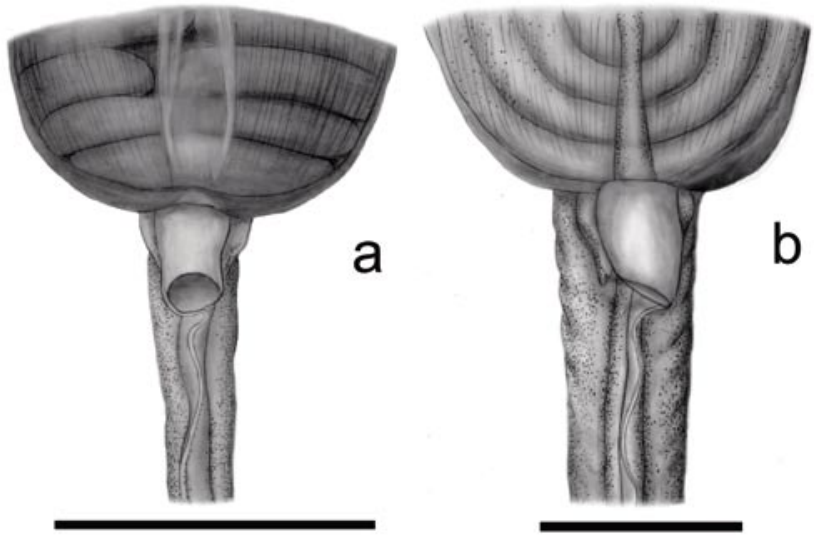

Figura 4. (a) Tubo ventral del renacuajo de Pleurodema marmorata. (b) Tubo ventral del renacuajo de Gastrotheca peruana. Barra de escala $=5 \mathrm{~mm}$. 
Tabla 2. Datos morfométricos para larvas de Telmatobius rimac. Excepto para los individuos en los estadios 32 y 35 , la primera fila representa la media \pm la desviación estándar y la segunda fila muestra el rango de las medidas. Longitud del cuerpo (LC), longitud total (LT), ancho del cuerpo (ANC), altura del cuerpo (ALC), diámetro del ojo (DO), distancia interorbital (DIO), distancia internarial (DIN), distancia hocico narina (DHN), distancia narina ojo (DNO), distancia hocico espiráculo (DHE), altura cola muscular en su inicio (ALCOM), altura máxima de la cola (ALCO), ancho de la cola muscular (ANCOM), ancho del disco oral (ANDO).

\begin{tabular}{|c|c|c|c|c|c|c|c|}
\hline Estadio & 27 & 28 & 29 & 30 & 32 & 35 & 36 \\
\hline $\mathbf{n}$ & 3 & 3 & 4 & 3 & 1 & 1 & 2 \\
\hline \multirow[t]{2}{*}{ LC } & $13,03 \pm 1,72$ & $15,82 \pm 1,09$ & $16,89 \pm 1,53$ & $17,15 \pm 0,66$ & 18,60 & 18,60 & $29,75 \pm 0,49$ \\
\hline & $11,10-14,40$ & $14,60-16,70$ & $15,80-19,10$ & $16,40-17,65$ & & & $29,40-30,10$ \\
\hline \multirow[t]{2}{*}{ LCO } & $25,03 \pm 2,64$ & $29,35 \pm 1,83$ & $30,25 \pm 1,67$ & $31,03 \pm 0,77$ & 34,50 & 37,30 & $49,25 \pm 0,21$ \\
\hline & $22,55-27,80$ & $27,45-31,10$ & $28,85-32,25$ & $30,15-31,55$ & & & $49,10-49,40$ \\
\hline \multirow[t]{2}{*}{ LT } & $37,95 \pm 4,49$ & $39,38 \pm 14,06$ & $47,68 \pm 3,33$ & $47,55 \pm 1,15$ & 52,05 & 54,40 & $76,38 \pm 1,87$ \\
\hline & $33,00 \pm 41,75$ & $23,15-47,90$ & $45,50-52,55$ & $46,40-48,7$ & & & $75,05-77,70$ \\
\hline \multirow[t]{2}{*}{ ANC } & $7,32 \pm 1,21$ & $8,50 \pm 0,30$ & $8,73 \pm 0,64$ & $9,10 \pm 0,58$ & 10,50 & 10,80 & $13,18 \pm 0,95$ \\
\hline & $6,05 \pm 8,45$ & $8,20-8,80$ & $8,05-9,60$ & $8,45-9,55$ & & & $12,5-13,85$ \\
\hline \multirow[t]{2}{*}{ ALC } & $5,37 \pm 0,70$ & $6,25 \pm 0,38$ & $6,6 \pm 0,06$ & $6,47 \pm 0,43$ & 7,20 & 7,05 & $13,28 \pm 0,95$ \\
\hline & $4,70 \pm 6,10$ & $5,85-6,60$ & $6,40-6,55$ & $6,05-6,90$ & & & $12,60-13,95$ \\
\hline \multirow[t]{2}{*}{ DO } & $1,33 \pm 0,06$ & $1,47 \pm 0,06$ & $1,58 \pm 0,10$ & $1,67 \pm 0,06$ & 1,80 & 1,90 & $2,40 \pm 0,00$ \\
\hline & $1,30-1,40$ & $1,40-1,50$ & $1,50-1,70$ & $1,60-1,70$ & & & 2.40 \\
\hline \multirow[t]{2}{*}{ DIO } & $3,80 \pm 0,53$ & $4,60 \pm 0,30$ & $4,63 \pm 0,22$ & $4,53 \pm 0,21$ & 5,60 & 5,20 & $7,65 \pm 0,64$ \\
\hline & $3,20-4,20$ & $4,30-4,90$ & $4,40-4,90$ & $4,30-4,70$ & & & $7,20-8,10$ \\
\hline \multirow[t]{2}{*}{ DIN } & $2,37 \pm 0,40$ & $2,97 \pm 0,23$ & $2,95 \pm 0,10$ & $2,63 \pm 0,20$ & 3,70 & 3,30 & $4,55 \pm 0,21$ \\
\hline & $2,00-2,80$ & $2,70-3,10$ & $2,90-3,10$ & $2,40-2,80$ & & & $4,40-4,70$ \\
\hline \multirow[t]{2}{*}{ DHN } & $2,10 \pm 0,40$ & $3,03 \pm 0,06$ & $2,88 \pm 0,10$ & $3,40 \pm 0,17$ & 3,40 & 2,90 & $4,75 \pm 0,21$ \\
\hline & $1,70-2,50$ & $3,00-3,10$ & $2,80-3,00$ & $3,20-3,50$ & & & $4,60-4,90$ \\
\hline \multirow[t]{2}{*}{ DNO } & $1,67 \pm 0,35$ & $2,20 \pm 0,26$ & $2,33 \pm 0,59$ & $2,17 \pm 0,12$ & 2,20 & 2,30 & $3,05 \pm 0,07$ \\
\hline & $1,30-2,00$ & $2,00-2,50$ & $1,90-3,20$ & $2,10-2,30$ & & & $3,00-3,10$ \\
\hline \multirow[t]{2}{*}{ DHE } & $7,38 \pm 1,20$ & $10,48 \pm 0,20$ & $10,61 \pm 0,77$ & $10,32 \pm 0,08$ & 12,65 & 12,30 & $16,55 \pm 0,35$ \\
\hline & $6,20-8,60$ & $10,25-10,60$ & $9,65-11,45$ & $10,25-10,40$ & & & $16,30-16,80$ \\
\hline \multirow[t]{2}{*}{ ALCOM } & $4,03 \pm 0,85$ & $5,32 \pm 0,28$ & $5,04 \pm 0,39$ & $5,77 \pm 0,12$ & 5,40 & 4,75 & $9,78 \pm 0,32$ \\
\hline & $3,40-5,00$ & $5,00-5,50$ & $4,45-5,30$ & $5,70-5,90$ & & & $9,55-10,00$ \\
\hline \multirow[t]{2}{*}{ ALCO } & $6,55 \pm 1,18$ & $7,53 \pm 0,65$ & $7,55 \pm 1,00$ & $8,57 \pm 0,28$ & 9,25 & 7,95 & $16,13 \pm 0,04$ \\
\hline & $5,30-7,65$ & $6,80-8,05$ & $6,10-8,30$ & $8,30-8,85$ & & & $16,10-16,15$ \\
\hline \multirow[t]{2}{*}{ ANCOM } & $2,47 \pm 0,45$ & $3,32 \pm 0,23$ & $3,31 \pm 0,19$ & $3,78 \pm 0,25$ & 5,60 & 4,20 & $7,08 \pm 0,04$ \\
\hline & $2,00-2,90$ & $3,10-3,55$ & $3,05-3,50$ & $3,50-3,95$ & & & $7,05-7,10$ \\
\hline \multirow[t]{2}{*}{ ANDO } & $4,18 \pm 0,87$ & $4,37 \pm 0,12$ & $4,29 \pm 0,17$ & $4,63 \pm 0,15$ & 5,45 & 5,35 & $8,55 \pm 1,63$ \\
\hline & $3,20-4,85$ & $4,30-4,50$ & $4,10-4,50$ & $4,50-4,80$ & & & $7,40-9,70$ \\
\hline
\end{tabular}

presente como una cresta. Abertura del tubo ventral sinistral. Altura máxima de la cola $5,30 \mathrm{~mm}$ y ubicada aproximadamente a mitad de su longitud, altura máxima de la cola muscular 3,40 $\mathrm{mm}$, ancho máximo de la cola muscular 2,00 mm. Disco oral de posición ventral, no emarginado (Fig. 2a). Papilas marginales presentes lateralmente en el labio anterior, y mental y lateralmente en el labio posterior. Papilas submarginales presentes lateralmente en el labio anterior y posterior. Envoltura mandibular dorsal más larga que la ventral, ambas con bordes ligeramente aserrados. Fórmula de filas labiales dentadas 2(2)/3.

Coloración: en líquido preservante, cuerpo tabaco grisáceo; cola muscular pardo cremosa, con pequeñas manchas tabaco esparcidas en toda su longitud; aletas dorsal y ventral claras, con pequeńas manchas tabaco, más abundantes en el tercio distal; vientre grisáceo claro, intestino visible.
Hábitat: No existen datos para los especimenes examinados, pero renacuajos de esta especie fueron encontrados en el río Sihua, tributario del río Cañete, a $3300 \mathrm{~m}$ de altitud, aguas abajo del pueblo Alis, Distrito de Alis, Provincia Yauyos (Mikael Lundberg observación personal). A esta elevación, el río Sihua tiene un ancho de aproximadamente ocho metros. Es un típico río altoandino con grava y piedras redondas de diferentes tamańos. En el cauce principal del río no existe vegetación; sin embargo, cerca a la orilla, existen zonas con menos corriente donde se puede encontrar plantas subacuáticas. Los renacuajos fueron encontrados en una zona con vegetación subacuática. Cuando fueron perturbados, los renacuajos se escondieron en la vegetación o en el fondo debajo de las piedras. En Ancash, renacuajos de esta especie fueron activos durante el día en partes con corriente lenta de arroyos y riachuelos (Lehr, 2001). 


\section{Clave para los anfibios del departamento de Lima Larvas}

No se incluye a Colostethus littoralis por carecer de muestras de su renacuajo.

1a. Disco oral no marginado (Fig. 2a).................................................. 2

1b. Disco oral emarginado (Fig. 2b-d).....................................................

2a. Hocico puntiagudo en vista dorsal (Fig. 3a)..... Telmatobius carrillae

2b. Hocico redondeado en vista dorsal (Fig. 3b)......... Telmatobius rimac

3a. Con papilas en el margen mental del disco oral (Fig. 2b)............ 4

3b. Sin papilas en el margen mental del disco oral (Fig 2 c, d)..............

4a. Tubo ventral medial (Fig. 4a), tercera fila del labio inferior de menor ancho que las dos filas más anteriores del mismo labio (Fig. 2b). ..................................

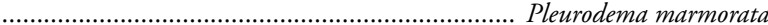

4b. Tubo ventral sinistral (Fig. 4b), tercera fila del labio inferior de aproximadamente el mismo ancho que las dos filas más anteriores del mismo labio. A.......................................................... Gastrotheca peruana

5a. Ancho del espacio en la segunda fila anterior de dentículos labiales es del 3 al 9\% del ancho de la misma fila; LTRF 2(2)/3[1] (Fig. 2c).

Bufo limensis

5b. Ancho del espacio en la segunda fila anterior de dentículos labiales es del 17

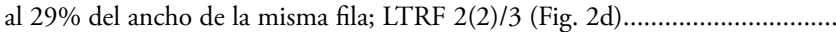

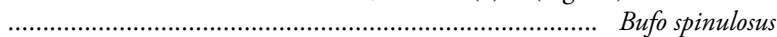

\section{Adultos}

1a. Glándulas parotoides presentes...................................................... 2

1b. Glándulas parotoides ausentes....................................................... 3

2a. Piel con o sin formaciones glandulares lenticulares o hemisféricas con proyecciones córneas múltiples, pero si presentes más abundantes lateral que dorsalmente................................................................ Bufo limensis

2b. Piel siempre con formaciones glandulares lenticulares o hemisféricas con proyecciones córneas múltiples, abundantes tanto lateral como dorsalmente................................................................................. Bufo spinulosus

3a. Parte terminal de los dígitos con discos expandidos...................... 4

3b. Parte terminal de los dígitos puntiagudo........................................ 5

4a. Parte terminal de los dígitos con dos escudos dorsales, hocico truncado en vista dorsal, hembras sin bolsa incubadora. ............ Colostethus littoralis

4b. Parte terminal de los dígitos sin escudos dorsales, hocico redondeado en vista dorsal, hembras con bolsa incubadora..................... Gastrotheca peruana

5a. Piel dorsal del cuerpo con glándulas redondas o alargadas. Pleurodema marmorata

5b. Piel dorsal del cuerpo sin glándulas visibles... ....6

6a. Dientes maxilares y vomerianos presentes, coloración ventral de los muslos anaranjado......................................................... Telmatobius rimac

6b. Dientes maxilares y vomerianos ausentes, coloración ventral de los muslos gris oscuro. Telmatobius carrillae

\section{Discusión}

En este estudio actualizamos la lista de reptiles y anfibios presentes en el departamento de Lima. Para este fin se tuvo en cuenta los registros bibliográficos mencionados en la introducción y los nuevos registros que fueron resultado de nuestro trabajo de campo. Sin embargo, algunas especies mencionadas en los listados anteriores no fueron incluidas dentro de la presente lista.

Stenocercus chrysopygus no fue incluida porque no conocemos ningún ejemplar de esta especie en las colecciones del MUSM y tampoco hemos encontrado ningún espécimen durante nuestro trabajo de campo.
Tabla 3. Lista de anfibios y reptiles registrados para el departamento de Lima. En la columna izquierda se muestra la clasificación tradicional y en la columna derecha se muestra una clasificación de anfibios que sigue a Frost (2007) y una de reptiles en base a la filogenia que presentan Pough et al. (1998) y Zug et al. (2001). Los nuevos registros para la herpetofauna del departamento de Lima están marcados con *

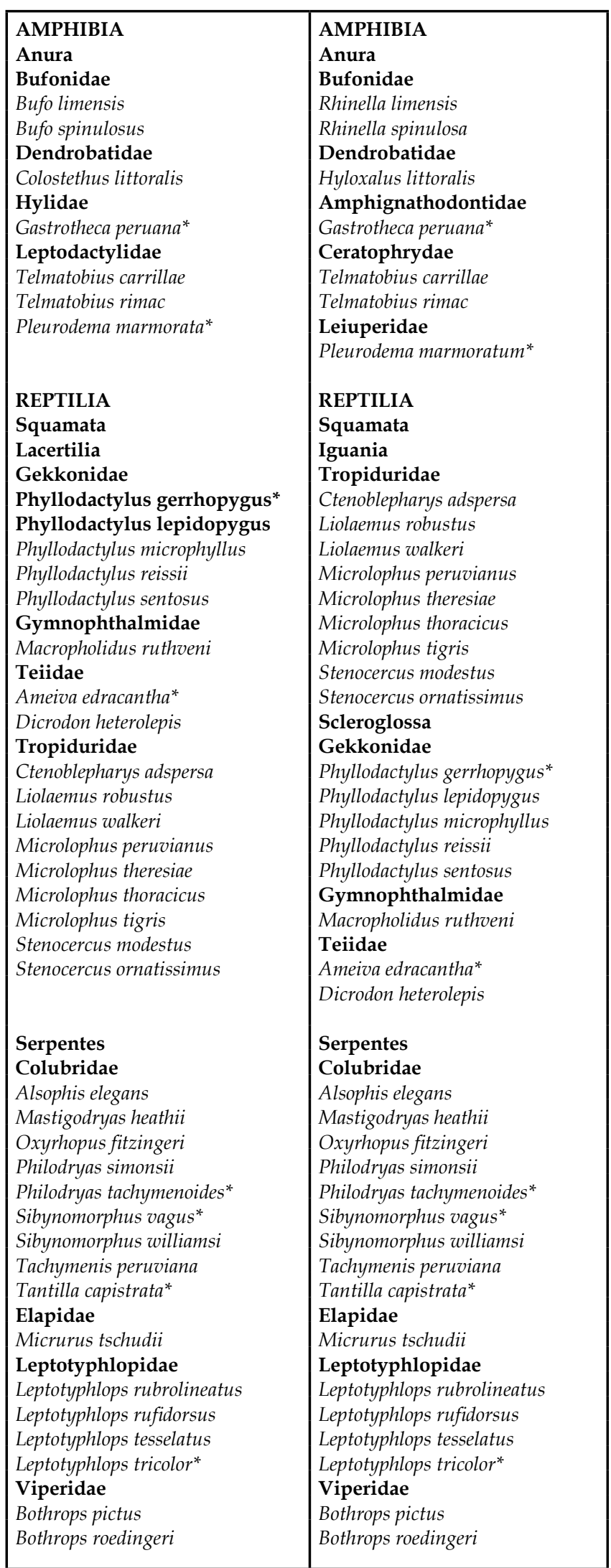


Tampoco incluimos a Microlophus koepckeorum que fue reportado por Henle y Ehrl (1991) en Ancón (distrito de la provincia de Lima) cerca del litoral costero en una localidad por debajo de los $100 \mathrm{~m}$ de altitud. Este registro es bastante dudoso debido a que $M$. koepckeorum tiene una distribución altitudinal conocida entre 285 y 2,100 m (Dixon y Wright, 1975). Creemos que existe una equivocación en la identificación del espécimen.

Del mismo modo Tropidurus arenarius (Tschudi, 1845) descrito en base a especimenes de Huacho (provincia de Huaura) tampoco fue incluido, porque desde su descripción no ha sido reportado ningún individuo de esta especie en el departamento de Lima. Creemos que la localidad típica mencionada en la descripción es errada, algo que no sería raro en las publicaciones de Tschudi. Igualmente Tachymenis elongata Despax, 1910 fue excluida de la lista para el departamento de Lima porque fue sinonimizada a Alsophis elegans (Tschudi, 1845) por Tipton (2005).

Por otro lado, la lagartija Liolaemus walkeri, reportada para Ticlio por Shrewe (1938), fue confirmada para la misma localidad durante nuestro trabajo de campo. Del mismo modo, Philodryas simonsii registrada por Thomas (1977) para Huarochirí, ha sido confirmada en varias localidades de departamento de Lima durante nuestras expediciones.

En la Tabla 3 se lista los anfibios y reptiles para departamento de Lima. Se presenta alternativamente una lista con la nueva clasificación de anfibios propuesta por Frost et al. (2006) y una de reptiles que sigue una clasificación coherente con las hipótesis filogenéticas propuestas para los escamosos (Pough et al., 1998; Zug et al., 2001; Townsend et al., 2004; Lee, 2005; Vidal y Hegdes, 2005). Aunque las relaciones de los escamosos son controversiales, las serpientes no están filogenéticamente separadas de las lagartijas y, por lo tanto, no se justifica los subordenes Lacertilia y Serpentes como dos grupos diferentes (Pough et al., 1998; Zug et al., 2001; Towsend et al., 2004; Lee, 2005; Vidal y Hegdes, 2005). Aquí seguimos la filogenia que se presenta en Pough et al. (1998) y Zug et al. (2001) que divide a Squamata en Iguania y Scleroglossa, estando las serpientes incluidas en este último grupo.

Este estudio incrementa para el departamento de Lima el número de anfibios y reptiles a 7 y 33 respectivamente. También proporcionamos información sobre la distribución geográfica de los anfibios y reptiles, y en particular facilitamos la identificación de los anuros elaborando claves para los individuos postmetamórficos y renacuajos presentes en el departamento de Lima.

\section{Agradecimientos}

A Jesús H. Córdova, Curador del Departamento de Herpetología del Museo de Historia Natural de la Universidad Nacional Mayor de San Marcos, que muy gentilmente brindó espacio y acceso a los especimenes. A Paúl Velazco por su apoyo en la obtención de literatura. El primer autor también agradece de manera especial a Alejandro Aguilar por su apoyo constante e invalorable en sus actividades académicas.

\section{Literatura Citada}

Altig, R. \& G.F. Johnston. 1989. Guild of anuran larvae: relationships among developmental modes, morphologies, and habitats. Herpetological Monographs. 3: 81-109.

Altig, R. \& R.W. McDiarmid. 1999. Body plan. Development and Morphology. En: McDiarmid, R.W. \& R. Altig, eds. Tadpoles. The Biology of Anuran Larvae. Chicago, the University of Chicago Press. 444 pp.
Aguilar, C. \& M. Lundberg. 2005. Geographic Distribution. Telmatobius carrillae. Herpetol. Rev. 36: 465.

Angulo, A. \& C. Aguilar. 2003. The Tadpole of Bufo limensis (Werner, 1901) (Anura: Bufonidae). Amphibia - Reptilia. 24: 400-405.

Cambell, J.A. \& W.W. Lamar. 2004. The Venomous Reptiles of the Western Hemisphere. Cornell University. Vol I: i-xvii, 1-476, [1]-[28], pl. 1-751; Vol II: i-xiv, 477-870, [1]-[28], pl. $752-1365$.

Carrillo de Espinoza, N. \& J. Icochea. 1995. Lista taxonomica preliminar de los reptiles vivientes del Perú. Publicaciones del Museo de Historia natural U.N.M.S.M. (A) 47: 1-27.

Dixon, J.R. \& R.B. Huey. 1970. Systematics of the lizards of the gekkonid genus Phyllodactylus of mainland South America. Los Angeles County Museum Contributions in Science. 192: $1-78$

Dixon, J.R. \& J.W. Wright. 1975. A review of the lizards of the iguanid genus Tropidurus in Peru. Nat. Hist. Mus. Los Angeles Contrib. Sci. 271: 1-39.

Donoso-Barros, R. 1966. Reptiles de Chile. Universidad de Chile. Santiago, Chile. $458+$ cxlvi.

Duellman, W.E. \& T.H. Fritts. 1972. A Taxonomic Review of the Southern Andean Marsupial Frogs (Hylidae: Gastrotheca). Occasional Papers of the Museum of Natural History of the University of Kansas Lawrence, Kansas. 9: 1-37.

Duellman, W. \& A. Veloso. 1977. Phylogeny of Pleurodema (Anura: Leptodactylidae): A Biogeographic model. Occasional Papers of the Museum of Natural History Museum the University of Kansas, Lawrence, Kansas 64: 1-46.

Frost, D. R. 2007. Amphibian Species of the World: an Online Reference. Version 5.1 American Museum of Natural History, New York, USA. <http://research.amnh.org/herpetology/ amphibia/index.php> (access 10 October, 2007)

Gosner, K.L. 1960. A simplified table for staging anuran embryos and larvae with notes on identification. Herpetologica. 16: $183-190$.

Henle, K. \& A. Ehrl. 1991. Zur Reptilienfauna Perus nebst Beschreibung eines neuen Anolis (Iguanidae) und zweier neuer Schlangen (Colubridae). Bonn. zool. Beitr. 42: 143-180.

Icochea, J. 1998. Lista roja preliminar de los anfibios y reptiles amenazados del departamento de Lima. En: Cano, A. \& K.R. Young, eds. Los Pantanos de Villa. Biologia y Conservacion. Museo de Historia Natural-UNMSM, Serie de Divulgación No 11, pp. 217-229.

Lee, M. S. Y. 2005. Squamate phylogeny, taxon sampling, and data congruence. Organisms, Diversity \& Evolution 5: 25-45.

Lehr, E. \& C. Aguilar. 2000. Geographic Distribution. Ameiva edracantha. Herpetol. Rev. 31: 52.

Lehr, E. 2002. Amphibien und Reptilien in Peru. Natur und Tier - Verlag GmBH, Münster. 208 pp.

Pefaur, J.E., A. Nuñel, E. Lopez \& J. Davila. 1978. Distribución y clasificación de los anfibios del departamento de Arequipa. Bull. Inst. Fr. Et. And. VII(1-2): 119-127.

Pesantes-Segura, O. 2000. Prevención y tratamiento de accidentes por serpientes venenosas. Centro Ecológico Recreacional Huachipa, Sedapal, Lima, Perú. 38 pp.

Peters, J., B. Orejas-Miranda \& R. Donoso-Barros. 1986. Catalogue of the Neotropical Squamata: Part I Snakes, Part II Lizards and Amphisbaenians, new material by P. E. Vanzolini. Smithsonian Institution Press. 1-26, v-viii, 1-347, 1-25, v-viii, 1-293.

Pough, F.H., R.M. Andrews, J.E. Cadle, M.L. Crump, A.H. Savitzky \& K.D. Wells. 1998. Herpetology. Prentice Hall, New Jersey. 577 pp.

Rodriguez, L.O., J.H. Córdova \& J. Icochea. 1993. Lista preliminar de los anfibios del Perú. Publicaciones del Museo de Historia natural U.N.M.S.M. (A) 45: 1-22. 
Shreve, B. 1938. A new Liolaemus and two new Syrrhopus from Peru. J. Washington Acad. Sci. 28(9): 404-407.

Tello, G. 1998. Lagartijas del Departamento de Lima, Perú. Biotempo 3: 59-63.

Thomas, R.A. 1977. A New Generic Arrangement for Incaspis and Mainland South American Alsophis and the Status of Two Additional Peruvian Species. Copeia 1977: 648-652.

Tipton, B.L. 2005. Snakes of the Americas: Checklist and lexicon. Krieger. 492 pp.

Townsend, T.M., A. Larson, E. Louis \& J.R. Macey. 2004. Molecular phylogenetics of Squamata: the position of snakes, amphisbaenians, and dibamids, and the root of the squamate tree. Syst. Biol. 53: 735-757.

Vellard, J. 1960. El género Pleurodema en los Andes peruanos. Memorias del Museo de Historia Natural 10: 1-12.

Walker, F.W. 1945. A study of the snake, Tachymenis peruviana Wiegmann and its allies. Bull. Mus. Comp. Zool. Harvard 96(1): $1-55,5$ plates
Vidal, N. \& B. Hedges. 2005. The phylogeny of squamate reptiles (lizards, snakes, and amphisbaenians) inferred from nine nuclear protein-coding genes. C. R. Biologies 328(10-11): 1000-1008.

Wassersug, R. \& W. Duellman. 1984. Oral Structures and their development in egg-brooding hylid frog embryos and larvae: evolutionary and ecological implications. Journal of Morphology 182: 1-37.

Wilson, L.D. 1999. Checklist and key to the species of the genus Tantilla (Serpentes: Colubridae), with some commentary on distribution. Smithsonian Herpetological Information Service. 122: 1-36.

Zeballos, H., L. Villegas, R. Gutiérrez, K. Caballero \& P. Jiménez. 2000. Vertebrados de las Lomas de Atiquipa y Mejía, sur del Perú. Revista de Ecología Latinoaméricana. 7(3): 11-18

Zug, G. 1977. Distribution and variation of Leptotyphlops tricolor. Copeia 1977: 744-745.

Zug. G.R., L. Vitt \& J. Caldwell. 2001. Herpetology. Academia Press, USA. $630 \mathrm{pp}$. 